\title{
La Relación del Derecho Internacional Privado con los Derechos Humanos. $^{1}$

\author{
The Relationship Between the International Private Law and the \\ Human Rights
}

\section{Leonel Pereznieto Castro}

Sumario: Introducción. II. Los derechos Humanos en el Derecho privado. III. Los Derechos Humanos en el Derecho Internacional Privado, A) Enfoque teórico. B) Dimensión práctica: a).Norma de Conflicto. b). Derecho Uniforme. IV. Conclusión.

\section{Resumen}

El presente trabajo se desarrolló mediante una metodología de transferencia de conceptos que permite romper el paradigma tradicional de los Derechos Humanos a fin de analizarlos en las relaciones entre particulares y con especial énfasis en el Derecho Internacional Privado.

\section{Abstract}

This work was developed through a methodology transfer of concepts that allows breaking the traditional paradigm of Human Rights to analyze relationships between individuals and with special emphasis on Private International Law.

\section{Introducción.}

La Reforma Constitucional de 2011 al Art. $1^{\circ}$ constitucional $^{3}$ fue la norma que impulsó el cambio de las garantías constitucionales - que han sido indispensables durante siglo y medio para combatir las arbitrariedades de la autoridad - a un concepto más amplio de protección de la persona que es el de los Derechos Humanos (DH) La exposición de

\footnotetext{
${ }^{1}$ El presente trabajo, fue originariamente una conferencia que el autor impartió en la Facultad de Derecho de la Universidad de Sonora el 19 de mayo de 2016

${ }^{2}$ Profesor de carrera T.C. en el Centro de Relaciones Internacionales de la Facultad de Ciencias Políticas de la UNAM e investigador Nacional Nivel III. Consultor del despacho Jauregui y del Valle. S.C. (Ciudad de México) Doctorado Honoris Causa de la Universidad de Sonora

${ }^{3}$ Diario Oficial de la federación 11. 06.2011
} 
este curso tiene que ver con una versión poco explorada de los $\mathrm{DH}$ que son los derechos privados y todavía dentro de esta clasificación, nos referiremos a la relación de los $\mathrm{DH}$ con el Derecho Internacional Privado (DIPr)

Un autor ampliamente conocido del DIPr, ya fallecido, el profesor argentino Werner Goldschmit, habló a finales de la década de los años cincuenta, del "Derecho a la Tolerancia" quizá por su experiencia personal como prófugo del nazismo que encontró refugio en España y más tarde el gobierno argentino generosamente, lo acogió y le otorgo su nacionalidad. La obra del profesor Goldschmit tiene constantes referencias en sus trabajos, al tratamiento del extranjero y puntualiza en el estudio, la necesidad de otorgarle los derechos plenos de persona que le permitan vivir dentro de un orden jurídico amigable y que ese estatus se proyecte a escala internacional. Con este fin dedicó en su tratado de DIPr de $1970{ }^{4}$ todo un capítulo al estudio de la persona y especialmente de sus derechos, cuando esa persona es extranjera, bajo el concepto del Derecho de la Tolerancia.

Sin embargo, este primer enfoque en el tema que tuvo muchos méritos en señalar cuándo y en qué circunstancias deben respetarse los derechos del extranjero ya ha sido ampliado considerablemente por los avances del concepto de los DH y de su vínculo con otras áreas que los han estudiado ampliamente, como es el caso de la filosofía, sociología y la ciencia política y especialmente por la universalidad con que se discuten hoy en día los $\mathrm{DH}$.

\section{Los Derechos Humanos en el Derecho Privado.}

Movámonos ahora hacia el tema específico de este trabajo, pero antes debemos definir el vínculo de los $\mathrm{DH}$ entre particulares. Aquí, como lo veremos más adelante, el cambio de paradigma se da de la forma siguiente: en la discusión tradicional de los $\mathrm{DH}$, el tema central se presenta en el ámbito de la defensa de esos derechos de la persona frente al Estado y en el caso de los $\mathrm{DH}$ en el derecho privado, es necesario definir el ámbito que cubre la protección del Estado ante las violaciones de $\mathrm{DH}$ entre particulares en las relaciones que estos desarrollan en la sociedad. Una cláusula contractual entre un distribuidor y una empresa transnacional dónde se ha pactado de manera jurídicamente formal que la terminación del contrato la pueden dar cualquiera de las partes en

4 Publicado en su décima edición actualizada por Alicia M. Peruguini Zaneti. Ed. Abeledo Perrot, Buenos Aires. 2011. 
cualquier momento, será una cláusula que viole los $\mathrm{DH}$ del distribuidor solo por la desproporcionalidad económica entre las partes. De ahí que resultará necesario defender una amplia serie de principios tales como la Certeza en la aplicación de la ley personal, donde quiera que la persona se encuentre, la Seguridad jurídica en las transacciones privadas; Que la desproporcionalidad económica entre las partes, no sea un factor que afecte a su necesaria igualdad contractual. Que las asociaciones o clubes no infraccionen a sus miembros con la suspensión del trabajo profesional: o bien, que se proceda con dolo en el comercio internacional, donde precisamente, la estructura que lo soporta, está basado en la buena fe de las partes contratantes, entre otros. En estas condiciones, no resulta una tarea fácil la definición de vínculo de los $\mathrm{DH}$ entre particulares dada su extensión y sobretodo, el casuismo que este planteamiento comporta. Hay razones que indican cierta dificultad para lograr una definición del vínculo entre $\mathrm{DH}$ y derecho privado. Una que veo como crucial es que se trata de un campo nuevo del derecho donde es necesario precisar cada una de las cuestiones que lo componen y determinar el ámbito que cubre la protección del Estado en estas relaciones; sin embargo, hay que iniciar la búsqueda de elementos que permitan la formación de un paradigma que nos lleve a una definición. La ventaja con que se cuenta en esta nueva dimensión jurídica es que se podrá alcanzar la formación de conceptos a partir del amplio casuismo que la compone; se trata de una disciplina que requiere de la uniformidad de criterios judiciales o arbitrales, que la vayan formando.

Por lo pronto hay que partir del ámbito que el Estado está dispuesto a cubrir con su protección. Un caso típico es la defensoría de los derechos del consumidor, dónde la presencia del Estado en los conflictos entre particulares ha servido de puente para encontrar soluciones que abonen en favor de la sociedad. Este es un ámbito dónde el Estado debe moverse con prudencia a fin de poder distinguir cuando hay una violación de los $\mathrm{DH}$ y cuando es una simple desavenencia de intereses entre particulares. El filtro lo serán los tribunales y podríamos estar ante la regulación de un ámbito del Derecho, con base en una formación jurisprudencial, que atienda más a las necesidades específicas de las personas, que lo que hace el legislador con una norma general. La formación de una norma de diferente origen del tradicional; es decir, de origen legislativo. En la nueva formación jurisprudencial que es probable se integre en materia de $\mathrm{DH}$ entre particulares, para la regulación de sus relaciones, se atiende a la necesidad específica de los individuos, cuando decidan en cada caso, cuándo y en qué 
medida se afecta a sus $\mathrm{DH}$, por lo que la labor que hagan los jueces en este ámbito, será decisiva.

Un destacado jurista colimense, Mario de Lamadrid Andrade, en defensa de la existencia de los $\mathrm{DH}$ en el derecho privado, se refiere a este tema como "la irradiación de los efectos de los Derechos Fundamentales hacia todo el ordenamiento jurídico incluido el derecho privado" El autor habla de los efectos que produce esa "irradiación" a partir de las disposiciones constitucionales que "representan un orden objetivo de valores que impregnan a la totalidad del sistema ...y por lo tanto reclaman...ser aplicadas sin intermediación en todos los sectores del Derecho." 5 Por su parte Lois Henkin, profesor de la Universidad de Columbia, ya fallecido, opinaba, desde una posición más abierta, que se trata de las libertades, inmunidades y beneficios que los seres humanos pueden reclamar conforme a "Los valores contemporáneos aceptados"6 Dado que hay que dar un paso más adelante en esta discusión, tratando de concretarla más, para lo cual necesario es hacer un primer deslinde respecto a la concepción de los $\mathrm{DH}$ : cuando hablamos de los $\mathrm{DH}$ frente al Estado o que el Estado protege en virtud de una convención, acuerdo o tratado internacional, estamos en una relación de orden vertical, en la medida que el Estado debe proteger toda violación provocada por alguno de sus funcionarios en contra de los $\mathrm{DH}$ de los particulares o incorporar la normatividad internacional en el orden jurídico nacional para aplicarla en un caso concreto; mientras que, cuando hablamos de la relación entre particulares, nos referimos a relaciones horizontales en las que se recurre al Estado solo en caso de conflicto y aún en esos casos, existen métodos que no requieren la presencia del Estado como son por ejemplo, la mediación y el arbitraje, que se pueden acordar a partir de la libre voluntad de las partes en el contrato mediante una prórroga de competencia judicial ${ }^{7}$. Así tenemos, principios que están impregnados de los "Valores contemporáneos" como los de la Buena Fe; la No afectación de Derechos de Terceros; la Libertad en la Contratación y la Autonomía de la Voluntad para la designación de la ley aplicable o para la prórroga de la competencia judicial en favor de los métodos alternos de solución

\footnotetext{
${ }^{5}$ De la Madrid, op.cit.

6 Human Rigths, en: Rüdiguer Bernhart, Encyclopedia of Public International Law, Vol.II. Amsterdam. Ed. North Holland, Elsevier, 1995, p.886

7 Art. 1051 del Código de Comercio, primer párrafo: "El procedimiento mercantil preferente a todos es el que libremente convengan las partes con las limitaciones que se señalan en este libro, pudiendo ser un procedimiento convencional ante tribunales o un procedimiento arbitral".
} 
de controversias, que entre otros, conforman ese arsenal de $\mathrm{DH}$ privados que tienen las personas entre sí y que deben ser ejercidos con la misma protección que les otorga el Estado a los derechos que se ejercen frente a él. De lo que se trata es de evitar, en la medida de lo posible la violación de los $\mathrm{DH}$.

Ahora bien, la forma de cobertura de los $\mathrm{DH}$ sobre las relaciones privadas ha sido descrita por la Corte Interamericana de $\mathrm{DH}$, en una resolución de $2016^{8}$ en los siguientes términos.

"Se determinó que la República de Costa Rica es internacionalmente responsable por haber vulnerado los derechos a la vida privada y familiar y el derecho a la integración personal en relación con la autonomía personal ( en razón de al) derecho a decidir si tener hijos biológicos a través de una técnica de reproducción asistida (También) a la salud sexual (y al) derecho de gozar de beneficios del progreso científico y tecnológico ( Así como al) principio de no discriminación"

Como puede verse, aquí la intromisión del Estado en una relación entre particulares (la mujer que solicitaba reproducción asistida y el médico que se la daría) dio lugar a una responsabilidad internacional por parte del Estado. Faltará ver cuando esa responsabilidad internacional se presente cuando el Estado haya intervenido para equilibrar o remediar una relación entre particulares.

De manera más concreta, en otra resolución de la misma Corte nos dice lo siguiente:

Que los principios de igualdad y no discriminación "Genera(n) efectos con respecto a terceros, inclusive a particulares (...) ya que sobre él descansa todo el andamiaje jurídico del orden público nacional e internacional y es un principio fundamental que permea todo el ordenamiento jurídico". Estos principios "representan un orden objetivo de valores que impregnan la totalidad del sistema...y por lo tanto reclaman...ser aplicados sin intermediación en todos los sectores del Derecho."

Se trata de una relación que surge entre particulares y que puede ser de distinto grado y naturaleza; sin embargo, hay casos específicos en los que los $\mathrm{DH}$ de las partes deben prevalecer, me refiero a derechos que ya señalé antes, tales como el respeto a los derechos adquiridos en el extranjero; a la aplicación de ley personal, no importa dónde se encuentre la persona; la Buena Fe; la Certeza jurídica; la Autonomía de la

\footnotetext{
${ }^{8} 26$ de febrero de 2016, caso Atravia Murillo y otros, supervisión de cumplimiento de sentencia.

${ }^{9}$ Opinión Consultativa 18/03, citada por De la Madrid,op.cit.
} 
Voluntad, entre otros, que deben ser considerados en las relaciones particulares y son a estas relaciones a las que nos referiremos en el presente curso.

\section{Los Derecho Humanos en el Derecho Internacional Privado.}

El tema de los DH es importante en el DIPr aunque está en sus inicios. Es más, en la formación de la disciplina, el tráfico jurídico internacional solo pudo ser una realidad cuando hubo libertad de movimiento de personas y bienes. Por su parte, la ley aplicable como de la jurisdicción competente y el acceso a la justicia, son los ejes de esa área. Los $\mathrm{DH}$, en estas condiciones, representan una constante, en la medida que se encuentran en la base de la protección del individuo, la familia y el comercio, a nivel global. Para lo cual un primer planteamiento sobre la cuestión hay que hacerlo desde el punto de vista teórico con objeto de deslindar el marco de referencia y desde la perspectiva práctica, para ilustrar las características concretas y efectos, de esos principios. Dado los límites de tiempo en un curso como el presente, se abordarán los grandes rasgos, en cada uno de los casos, procurando enfocar algunos de sus respectivos aspectos relevantes.

\section{A. Enfoque Teórico.}

Uno de los filósofos del Derecho actuales más importantes, con una mirada fresca hacia la Sociología, es Robert Alexy, junto con otros autores contemporáneos, representa gran parte del pensamiento europeo actual, que se ha dedicado a estudiar al individuo en su interactuación de sus relaciones sociales, desde diferentes ópticas a partir de una determinada construcción social. Así "La realidad no es algo externo al conocimiento sino es constituido por el conocimiento en sí", como es el caso de un post- constructivista, como Focault ${ }^{10}$ ) o quien propone, desde una postura crítica, como Jurgen Habermas ${ }^{11}$, una teoría a partir de la comparación directa del discurso teóricoempírico de las ciencias con el discurso práctico normativo de la política, la moral y el derecho, donde "La validez de sus declaraciones depende de la corrección del

10 Politics, Philosophy, Culture: Interviews and Other Writings, 1977-1984. Ed. Lawrence D. Kritzman, Dartmuoth College, New Hampshire.1988

${ }^{11}$ Teoría de la Acción Comunicativa, 2 vols. Ed. Trotta, Madrid, 2007 
procedimiento" y Alexy que nos brinda un aparato conceptual muy amplio e interesante sobre este tema, en su obra fundacional: "Teoría de los Derechos Fundamentales ${ }^{12 " ~ y ~}$ en toda su demás amplia obra publicada en libros y en especial, en revistas especializadas $^{13}$. En su obra Alexy, se refiere a los Derechos Humanos y su defensa y sobretodo, lo hace bajo un enfoque actual. El autor distingue cuatro propiedades de los $\mathrm{DH}$, la primera, la de su Universalidad, porque su titularidad corresponde a todo ser humano; una segunda, es su Validez Moral porque se trata de derechos morales. Una tercera cualidad es su "Fundamentalidad" que concierne al objeto de los derechos y su cuarta característica es su Prioridad frente al derecho positivo ${ }^{14}$

Conforme a las ideas de Robert Alexy, expresadas principalmente en su Teoría de los Derechos Fundamentales, el análisis que desarrolla el autor en forma comprensiva, es el estudio de los Derechos Humanos. Se trata, nos dice, de "La consideración sistemático-conceptual del derecho válido" en su dimensión empírica, de amplio espectro y que no se limita a los conceptos de derecho o de validez del derecho positivo, sino también a la búsqueda de la eficacia del derecho sin la cual, no se puede hablar de defensa alguna de los Derechos Humanos. Esa defensa se da "En la medida en que es condición de la validez positiva del derecho legislado y judicial [y que por tanto constituye el] objeto de la dimensión empírica."

Hay un par de comentarios que pueden hacerse a este pasaje de la obra de Alexy. En primer lugar la frase "La consideración sistemático-conceptual del derecho válido". De la lectura de su obra, se puede encontrar que los Derechos Humanos son el objeto directo de estudio y protección por parte del Estado y en esa medida, esta protección se integra a la sistemática del Derecho y se convierte así en el concepto del Derecho válido, ese que toma valor por el solo hecho de la protección y además, porque se trata

\footnotetext{
12 Ed. Centro de Estudios Constitucionales. Madrid. 2007.

${ }^{13}$ Como es el caso de sus obras la Ciencia de la Sociedad (Traducida al español por Javier Torres Nafarrete y publicada por la Universidad Iberoamericana y el ITESO. 1996) que junto con su Introducción a la Teoría de Sistemas, constituyen dos obras claves en el estudio de la metodología interdisciplinaria moderna. La Teoría de los Derechos Fundamentales, originalmente publicada como tesis de Doctorado en 1985, fue publicada en español por el Centro de Estudios Políticos y Constitucionales de Madrid, en 2002.

14 Alexy, R, La Institucionalización de los derechos humanos en el Estado Constitucional democrático, en: Revista de Derecho Constitucional, N 66. Año 22. Septiembre-diciembre 2002, pág, 21 y sigs.
} 
de un valor que está incorporado en todas las normas del sistema, "En la medida en que es condición de la validez positiva del derecho legislado y judicial, que por tanto constituye el objeto de la dimensión empírica". Es en esta dimensión, dónde el autor plantea la legitimidad o la legitimación del derecho válido. Alexy también nos dice: que una concepción como la que plantea "No se limita a los conceptos de derecho o de validez del derecho positivo jurídico" el derecho que Alexy plantea, sale de los estrechos límites del positivismo en "La búsqueda de la eficacia del derecho sin la cual, no se puede hablar de defensa alguna de los Derechos Humanos". Finalmente, la dimensión empírica a la que se refiere Alexy, es, como se sabe, la dimensión más rica para el DIPr, porque es ahí donde natural y finalmente, se expresa la disciplina en términos tales como:¿en qué medida se respeta la ley personal del individuo o los derechos adquiridos por él? ¿Cuántos juicios con elementos extranjeros se han resuelto?, ¿Cómo han sido resueltos?, ¿Qué tanto se respetaron efectivamente los derechos de las partes?, ¿Hubo un Debido Proceso? y, las sentencias, ¿Qué calidad tienen?

De esta manera, intentaremos ampliar el análisis del DIPr para que en su interpretación, se incluyan principios modernos en la disciplina, pero sobre todo, principios que protejan los $\mathrm{DH}$ de los particulares. En este sentido, retomemos algunas ideas más de Robert Alexy para enriquecer el análisis.

Volvamos en el tema que nos ocupa, a uno de sus ejes centrales: "La consideración sistemático-conceptual del derecho válido". En la línea de análisis del autor, el concepto de Norma de Derecho Fundamental plantea una serie de cuestiones compatibles con la normativa propia del DIPr, en relación con el tema de los Derechos Humanos y nos brinda soluciones interesantes. Alexy, nos dice: "Siempre que alguien posee un derecho fundamental, existe una norma válida de derecho fundamental que le otorga ese derecho". Detengámonos un momento para clarificar la idea con un ejemplo: un $\mathrm{DH}$ se encuentra consagrado en un tratado internacional no ratificado por México. De acuerdo al Art. $1^{\circ}$, primer párrafo de la Constitución, "En los Estados Unidos Mexicanos todas las personas gozarán de los derechos humanos reconocidos en esta Constitución y en los tratados internacionales de los que el Estado Mexicano sea parte..." Sin embargo, ese derecho existirá - según Alexy - en la medida que una norma válida consagre ese derecho fundamental. Con independencia de que México haya ratificado o no el tratado. Estamos en presencia entonces, de la protección que 
tienen todas las personas para que se les respeten sus $\mathrm{DH}$ ya que la validez de esos derechos deriva de una norma internacional válida - el tratado - que creó una norma fundamental que ya ha sido reconocida internacionalmente y por tanto debe aplicarse internamente.

El punto de partida de la propuesta de Alexy es "El concepto semántico de norma" y la distinción entre norma y enunciado normativo que en nuestro tema sería la definición de los $\mathrm{DH}$. En este sentido, Alexy hace una propuesta diferente que en breve consiste en que toda norma se expresa a través de "el significado de un enunciado normativo"; es decir, además de lo previsto en el enunciado, que puede ser una orden, un mandato o una permisión, esa norma tendrá siempre un valor incluido en el texto del enunciado - por ejemplo, la definición de los DH que finalmente será la que sostiene a la norma en la medida que es el valor que espera la sociedad que se cumpla, por tratarse de un $\mathrm{DH}$. A este respecto, nos dice el autor "el concepto de norma es el concepto primario con respecto al concepto de enunciado normativo" cuya validez la otorga la autoridad legalmente capacitada, Alexy sostiene también: "En la medida en que se menciona la imposición por parte de una autoridad autorizada por una norma de grado superior, puede hablarse de una Teoría Jurídica de Validez"

Alexy se refiere a lo que él llama "La Norma de Derecho Fundamental" o norma "lusfundamental". Respecto a ésta nos dice: se trata de las "Normas de derecho fundamental expresadas a través de disposiciones iusfundamentales, normas que por otro lado, se ubican como iusfundamentales de forma exclusivamente, como enunciados en las leyes primarias o en las constituciones".

Así para el DIPr podemos hablar de disposiciones iusfundamentales en la medida que se trata de normas o instituciones jurídicas reconocidas en la gran mayoría de países y que representan los derechos que llevan las personas consigo, no importa a dónde, cuándo y cómo se trasladen de un país a otro. Los derechos "lusfundamentales" deben estar siempre con la persona para su protección; como son los derechos a la Buena Fe y el de Certeza, con los que la persona celebra sus transacciones internacionales. Estos derechos son los que tratan de preservar la norma de conflicto y la uniformidad de derechos. Veamos ambos casos. 


\section{a). Norma de conflicto.}

A este propósito me refiero al trabajo en proceso de Jurgen Basedow del Max Planck Institut, para su informe al Instituto de Derecho internacional. ${ }^{15}$ Entre las propuestas que este autor hace podemos mencionar dos de ellas:

"Los estados respetan los derechos del hombre a través de sus órganos incluyendo sus jurisdicciones ordinarias dentro de las relaciones internacionales de personas privadas. Esos derechos sirven para controlar y corregir en caso dado, los resultados obtenidos por la operación de las reglas de conflicto"

Operación en la que diferentes puntos de vista coinciden que en efecto, la norma de conflicto debe ser un filtro para preservar esos valores sobre los cuales se encuentra asentada la sociedad. Pero igual de importante, el DIPr. será a través de su Norma de Conflicto un instrumento de control y de corrección en los resultados con motivo de aplicar DH en el tráfico jurídico internacional.

La segunda propuesta del profesor Basedow, respecto a las normas de conflicto es como sigue:

"La interpretación de las reglas de conflicto ya sean nacionales o las adoptadas por una organización de integración económica regional deben tener en cuenta la armonización entre los derechos del hombre, aplicables"

Vista la regla de conflicto desde esta perspectiva, la opción del juez dejará de ser entre "El conflicto" que le presentan dos leyes susceptibles de ser aplicadas y se circunscribirá a buscar el equilibrio con la integración de dos normas de $\mathrm{DH}$ en presencia, a fin de que su aplicación resulte adecuada para lo cual, el juez debe llevar a cabo una labor de integración de dichas normas. "La armonización entre los derechos del hombre, aplicables" a que se refiere Alexy.

\section{b). Derecho Uniforme.}

Pasemos ahora, al Derecho Uniforme, aquí como lo veremos más adelante en los ejemplos que analizaremos, el esfuerzo está dirigido para facilitar las condiciones de tráfico jurídico internacional y por tanto de vida de las personas con el respeto y protección de su Estatuto Personal o proteger el ejercicio de la Autonomía de la

15 En este sentido agradezco las gestiones del Dr. José Carlos Fernández Rozas, catedrático de la Universidad Complutense de Madrid por haberme hecho llegar los documentos preparatorios de la Comisión $\mathrm{N}^{\circ} 4$. Droits de l'homme et droit International privé, documento de trabajo, marzo de 2015. 
Voluntad y el principio de Buena Fe con objeto de que la persona pueda realizar transacciones internacionales.

Con estas propuestas en su conjunto, podemos ahora analizar la reforma al segundo párrafo del nuevo Art. $1^{\circ}$ constitucional cuando nos dice:

La Constitución y los tratados internacionales, se interpretarán en materia de $\mathrm{DH}$ "Favoreciendo en todo tiempo a las personas la protección más amplia" Protección que es una directriz que deben de acatar, las normas de conflicto, sobre todo cuando se trate de un derecho humano.

Respecto de la naturaleza del vínculo entre los Derechos fundamentales y el DIPr. Se puede responder que la semejanza normativa entre los $\mathrm{DH}$ y el DIPr. dimana de tres puntos precisos:

i) la reglamentación de las relaciones jurídicas de las personas a nivel internacional y el respeto a sus derechos adquiridos;

ii) el respeto irrestricto a la autonomía de la voluntad incluyendo, libre designación de la ley aplicable y prórroga, si es el caso, de la competencia judicial.

iii) el derecho que se tiene ante el juez de que éste respete aplique la ley personal del individuo, la ley que las partes han escogido para su contrato y los derechos de las personas en sus actividades de comercio;

\section{B. Dimensión Práctica}

Con estas ideas en mente, pasemos ahora a la dimensión práctica, que en la línea de argumentación que estamos siguiendo, corresponde a los efectos de la vinculación del DIPr. con los DH. Para lo cual propongo estudiar tres grupos de casos:

a) Normas jurídicas internacionales aplicables a los nacionales o domiciliados en territorio nacional.

b) El deber de aplicar el Estatuto Personal con objeto obtener la ley extranjera aplicable y en especial, cuando se trate de los derechos de unión y permanencia familiar y otros derechos adquiridos,

c) El respeto a la irrestricta autonomía de la voluntad para escoger la ley aplicable, la jurisdicción competente y en su caso la prórroga de la competencia judicial hacia medios alternativos de solución de controversias, en especial la Medición y el Arbitraje. Veamos brevemente cada uno de estos casos. 
En cuanto al primero, la aplicación de normas internacionales para los nacionales y domiciliados en México. Con objeto de abreviar la idea, permítanme dar un ejemplo, en el caso de México.

Vía Art. 133 constitucional, la norma jurídica internacional se incorpora al sistema jurídico nacional como norma de máxima jerarquía. Por otro lado, los tratados o convenios internacionales están elaborados para facilitar el tráfico jurídico internacional y elevar el nivel de los derechos de las personas que pertenecen a los países que firman el tratado. Sin embargo, la incorporación de un derecho más favorable en el sistema jurídico interno, especialmente en materia de $\mathrm{DH}$, siempre debe subsistir dentro del sistema, ya que la norma jurídica internacional se "nacionalizó" y se integró al orden jurídico interno ${ }^{16}$ y con la ampliación de su interpretación "Favoreciendo en todo tiempo a las personas la protección más amplia" conforme el segundo párrafo del Art. 1 constitucional antes citado, la aplicación de esa norma de Conflicto puede llevarse a cabo.

Ciertamente estamos en un tema poco explorado, al menos de la perspectiva del DIPr. Sin embargo, partimos del principio del "Bienestar Común", que ha sido objeto de la jurisprudencia en materia de Derechos Humanos, al menos en el continente latinoamericano ${ }^{17}$. Se trata que el legislador interno o el juez, se supediten a las normas internacionales en materia de Derechos Humanos que propendan al Bien Común. Una norma válidamente creada a nivel internacional por un órgano, la Comisión interamericana de $\mathrm{DH}$ de la que México es Estado Parte. Además, el contenido de una norma de esta naturaleza, por lo general, establece derechos más específicos, nuevos derechos o simplemente, regulaciones que facilitan el alcance de esos derechos.

Primer grupo, el grupo a). Antes propuesto: Normas jurídicas aplicables a personas domiciliadas en México: un ejemplo sobre la Adopción Internacional

El ejemplo que nos ayudará a precisar este concepto, es el de la Adopción Internacional. Tal y como está prevista en los tratados más importantes ratificados por

16 Pereznieto, L., El Art.133 constitucional, una relectura, en: Revista Jurídica. Universidad Iberoamericana. N²5,1994.

17 Sobre este tema, véase García Ramírez, S. y Morales Sánchez, J., La reforma constitucional sobre derechos humanos (2009-2011), México, Porrúa-UNAM, 2011. 
México $^{18}$. En éstos tratados, por lo general, se establecen procedimientos más expeditos que los establecidos localmente. Partamos para nuestro ejemplo de una persona domiciliada en México que desea usar dicho mecanismo de facilitación, o bien, extranjeros domiciliados en México, que desean adoptar niños mexicanos mediante un procedimiento simplificado y con ello alcanzar el Bien Común para que el menor huérfano mexicano, tenga una familia y crezca al lado de ésta. Pero sucede que el tratado está elaborado a fin de que extranjeros con origen en los países que han ratificado la convención puedan tener acceso al procedimiento de facilitación que ofrece el tratado, lo que también podría ser una limitante para el mexicano domiciliado en México.

Veamos los dos casos planteados, primero el mexicano domiciliado en México a quien, en estricto sentido no le es aplicable la Convención ya que ésta se encuentra formulada para adopción de menores mexicanos por parte de extranjeros domiciliados en el extranjero y en el segundo caso, el del extranjero domiciliado en México, que no tiene acceso tampoco al procedimiento de facilitación, simplemente por el hecho de que su Estado de origen, no ha firmado el tratado o convención internacional y por tanto ya no tendría acceso al ejercicio de ese $\mathrm{DH}$.

El precepto constitucional reformado al que hemos hecho mención, nos dice que cuando se trata de $\mathrm{DH}$, se le debe otorgar a la persona, la protección más amplia y en el caso que nos ocupa, la facilitación para el ejercicio del derecho de adopción, debe ser igualmente amplio, en cuyo caso cabría la posibilidad que ese mexicano pueda tener acceso al mecanismo de facilitación. No hay que desconocer, sin embargo, que el ejercicio de este $\mathrm{DH}$ está vinculado con un tema distinto, de carácter instrumental. Pero no menos importante. Un convenio internacional implica necesariamente obligaciones para los Estados firmantes del tratado que son indispensables para su funcionalidad.

Puede ser este último un argumento de peso pero es compensable con una estructura efectiva de supervisión y de compromiso sobre el seguimiento que se le debe dar al menor adoptado. En las convenciones internacionales se establece la obligación para el Estado parte, de darle seguimiento al cuidado de los padres adoptivos sobre hijos

\footnotetext{
${ }^{18}$ El interamericano sobre Conflictos de Leyes en materia de adopción de menores (1992) y el celebrado en la Conferencia Permanente de la Haya de DIPr. Sobre adopción internacional (1998)
} 
adoptivos, en el proceso de acoplamiento familiar, en la práctica es hasta de dos años. Este que es un procedimiento administrativo de supervisión temporal y puede ser materia de acuerdo entre las autoridades mexicanas con la persona que desee adoptar por la vía de facilitación. Ya sea que la persona esté domiciliada en México en cuyo caso deberá informar al DIF local o bien, si su domicilio está en el extranjero, informar al consulado mexicano de la localidad y permitir visitas de dichas autoridades. Ciertamente no es un procedimiento que pueda convertirse en ejecutivo a fin de separar a un menor adoptado y que es maltratado, de su familia de adopción o peor aún, en aquellos casos, afortunadamente menos hoy en día, en los que la adopción sirva para introducir al menor en una red de prostitución. En realidad, para evitar esos contratiempos el juez dispone actualmente de la información completa de cualquier persona, incluyendo, si los hubiere, sus antecedentes penales, a fin de poder distinguir con claridad que la persona de los adoptantes sea adecuada para la adopción. Además, es importante mencionar, que los expedientes de adopción los preparan minuciosamente autoridades del DIF especializadas (Sicólogos, personas de trabajo social, médicos, etc.) que durante meses previos a la adopción, observan la compatibilidad del menor susceptible de ser adoptado con los futuros adoptantes, con lo que el riesgo de que el menor adoptado sea maltratado o dedicado a otros fines distintos de la adopción, es altamente improbable.

He puesto ese ejemplo obviamente de manera muy general ya que el tema envuelve cuestiones más complejas, como el de la adopción por extranjeros no domiciliados en México procedentes de países que no son parte de la Convención o los casos de doble jurisdicción, etc., pero lo importante es ilustrar cómo un mecanismo facilitador puede ser el mejor conducto para alcanzar el Bien Común de un menor huérfano mexicano. Por otro lado, no debe estar sujeto a duda, si a un mexicano se le aplica una norma fundamental en su propio orden jurídico por el solo hecho de que la Convención únicamente esté dispuesta para extranjeros domiciliados en el extranjero o cuando un extranjero domiciliado en México, esté sujeto a la firma del tratado, por su Estado de origen. La norma jurídica internacional, al incorporarse al sistema jurídico mexicano, ya lo mencionamos, al "nacionalizarse", debe servir para todas las personas que se encuentren dentro de territorio nacional o quienes quieran adoptar a un menor, contraer matrimonio o celebrar un contrato, por una vía de facilitación que le pueda dar una convención internacional. 
La misma cuestión que la anterior, vista desde otro ángulo y la menciono para una posible discusión posterior; se trata de lo siguiente: se puede decir que el ámbito personal de aplicación del tratado no incluye a un mexicano o a un extranjero domiciliado en México y por tanto no existe un procedimiento específico para estos; sin embargo, un procedimiento que mejora los derechos de la persona, en este caso, un $\mathrm{DH}$ y se trata de una norma internacional se incorporó al derecho nacional, en mi opinión no puede ser excluyente con respecto a ninguna persona que tiene su domicilio en México. Dicho en otros términos, por el hecho de la incorporación de la norma jurídica internacional al sistema interno, perdió el ámbito de aplicación personal que le da el tratado y al ser norma nacional incorporada es aplicable a todas las personas que se encuentren domiciliadas dentro de territorio nacional, por ser en favor de los $\mathrm{DH}$ de cada persona. Se tratará de una elevación de la norma interna al nivel de la norma internacional correspondiente, en materia $\mathrm{DH}$ que será pues la vía para alcanzar el Bien Común en ambos casos que es la adopción del menor huérfano mexicano y que éste tenga una familia.

Veamos el segundo grupo, el

\section{b). Estatuto personal.}

Aquí partimos del deber del juez mexicano de aplicar el estatuto personal del individuo y máxime cuando se refiere a la unión familiar.

Respecto de este principio se trata obviamente de una idea muy clara; sin embargo, con frecuencia, los jueces mexicanos no lo aplican por ignorancia, por indiferencia o simplemente por no complicarse la vida en el procedimiento. De ahí la necesidad de promover el conocimiento del DIPr. entre los jueces del país para contribuir a que se cumpla con este derecho fundamental de las personas.

Al mismo tiempo, en la medida que los jueces respeten el estatuto personal del individuo, estarán dándole cumplimiento a un derecho fundamental de la persona que es aplicar su ley en beneficio de su seguridad y certeza jurídicas. Ejemplos son, el reconocimiento de un matrimonio celebrado en el extranjero o la adopción, celebrada en el extranjero, lugar de un menor cuyos derechos se pretenden ejercer en México, por ejemplo, para participar, por ejemplo, como heredero en una sucesión. 


\section{c). Autonomía de la Voluntad.}

A continuación, me refiero al último caso, el respeto al uso irrestricto de la Autonomía de la Voluntad. Aquí me propongo mostrar a través de un ejemplo cómo la Autonomía de la Voluntad debe ser protegida y a continuación, me referiré a unas palabras del profesor Eric Jayme, destacado profesor en la Universidad de Heildelberg sobre el tema que nos ocupa.

En cuanto al ejemplo, permítanme presentar un ejemplo un poco más complicado pero que muestra los puntos finos en materia contractual:

Una empresa transnacional fabricante de automóviles celebra contratos con sus distribuidores y en esos contratos se establece en la cláusula de vigencia y denuncia del contrato, vigencias cortas, de un año que mantienen en vilo al distribuidor y además, la facultad de la empresa de declarar terminado el contrato en cualquier momento, notificando al distribuidor con 30 días de anticipación, independientemente que el derecho del distribuidor sea el mismo que el del fabricante, para dar por concluido el contrato, porque el fabricante es la parte con poder real en la relación contractual. La empresa da por concluido el contrato tres meses después de la última celebración contractual por décimo año consecutivo. Aquí claramente hay una violación al $\mathrm{DH}$ del distribuidor. Si bien la cláusula es formalmente equilibrada y conforme a derecho, no lo es en la realidad ante dos partes tan desiguales y con base en esa desigualdad, la parte más fuerte en la relación contractual abusando de su fuerza, puede declarar la terminación cuando el distribuidor se prepara por décimo año consecutivo a volver a invertir en una nueva plantilla de vendedores, su entrenamiento, inversión en los talleres para ofrecer un mejor servicio de mantenimiento a los vehículos, etc. Ese desequilibrio provoca una violación a los $\mathrm{DH}$ del distribuidor que el juez o el árbitro deben hacer valer por encima de la formalidad del derecho. Eso es justicia. Este ejemplo nos ayudará a entender el planteamiento que sigue.

El profesor Eric Jayme nos dice: EI DIPr. tiene el deber de proteger a las personas frente a un mundo globalizado, en un doble sentido, primero, que esa persona pueda siempre, de acuerdo a su voluntad, resolver los asuntos que plantea en ese mundo globalizado conforme a los principios de seguridad y certeza y que no se encuentre con sorpresas desagradables, en nombre de la aplicación de normas de aplicación 
inmediata o de orden público, que trastocan el entendimiento que tuvo la persona al escoger como aplicable a sus relaciones jurídicas internacionales, una determinada ley $^{19}$.

En segundo término y no menos importante consiste en que la Autonomía de la Voluntad pueda llevar a esa persona a someterse a un tribunal previamente escogido y que no, el juego de la norma conflictual en materia jurisdiccional, lo lleve a acabar frente a un tribunal que no había sido previsto en sus relaciones internacionales. Dicho en otros términos, respetar y proteger lo que otro prestigiado profesor suizo, Alfred von Overbeck señaló a principio de la década de los noventa como: "La irresistible extensión de la autonomía en el DIPr." 20

No todos los casos son tan evidentes como los ejemplos al que nos referimos, cuando se trata de transacciones comerciales, en éstas, hay un ingrediente importante para la igualación de derechos a fin de que las transacciones comerciales sean justas para las dos partes. Veamos un ejemplo. La Convención de Naciones Unidas sobre Contratos de Compraventa de Mercancías (1980) establece reglas que definen derechos y facilitan su ejecución. Lo mismo ¿Cómo negarlas a una persona procedente de un país no miembro del tratado? Hacerlo sería situar a la persona en una situación de desventaja. Por el solo hecho de que México decidió incorporar a su sistema jurídico un sistema internacional de compraventa de mercancías avanzado. El sistema jurídico, se ha modernizado en ese sector, y éste debe ser para beneficio de todas las personas. Un caso solamente podría justificar la excepción a esta propuesta y consiste en lo siguiente:

Es el caso de los tratados comerciales, fiscales y otros que requieren una interactuación mutua y permanente de los Estados signatarios del tratado. Además obedecen a una política internacional muy definida. Sólo con tales o cuales Estados, le conviene al Estado Mexicano otorgar preferencias arancelarias a cambio de otras

\footnotetext{
${ }^{19}$ L'application par le juge interne des conventions du droit international privé.Recueil des Cours de la Academie de Droit International Privé, 1971. I. Pág. 99

${ }^{20}$ VON ÜVERBECK, A.E., 'Tirresistible extension de l'autonomie en droit international privé", Hommage Frar!fois Rigaux, Bruselas, 1993, pp. 619-636;
} 
tantas y con otros Estados no. Pero aún en este ámbito, un derecho sustantivo vinculado a los Derechos Humanos de la persona, deberá ser extensivo para todas las demás. No encontramos una razón jurídica para excluirlo.

En breve, ojalá que más trabajos se incorporen al desarrollo de los Derechos Humanos dentro del campo del DIPr. Una forma de asegurar la aplicación de la ley correcta, es que los jueces entiendan que se trata de una normatividad que trae implícita la eficacia a que se refiere Alexy. Esos valores iusfundamentales en que debe reposar cualquier disposición, sobretodo porque se tratará de Derechos Humanos básicos.

\section{Conclusión}

Ojalá que estas reflexiones motiven a algunos que desean encontrar un campo virgen en el estudio del DIPr. Su parte sustantiva debe desarrollarse comenzando por definir el acento que debe tener cualquier norma, en el valor incluido en el enunciado como lo propone Alexy. Esas normas mediante las cuales relaciones jurídicas familiares, personales y transacciones jurídicas en general pueden tener valor jurídico no importa donde la persona se encuentre.

\section{Material bibliográfico:}

\section{A. Doctrina}

AGUILAR, María Virginia, Un nuevo impulso a la Restitución Internacional de Menores y la Aplicación de la Convención de La Haya sobre los aspectos Civiles de la sustracción internacional de menores en México, en RMDIPyC. N26. Junio de 2010. Pág.121

- $\quad$ Y con RENDÓN, Roberto y PEREZNIETO CASTRO Leonel, Comentarios a la sentencia de 16 de febrero de 2009 emitida por la Tercera sala del Tribunal Superior de justicia del Distrito federal sobre sustracción ilegal de menores, en RMDIPyC. Octubre de 2009. pág. 103.

AZCÁRRAGA MONSONIS, Cármen. Sociedad Multicultural. Hacia una conformación novedosa del concepto de orden público internacional, en RMDIPyC. N²5 Octube de 2009

BERCOVITZ RODRIGUEZ Cano, Hijos made in California, Aranzadi, España. 
CALVO CARAVACA, A.L./CARRASCOSA GONZÁLEZ, J., Gestación por sustitución y derecho internacional privado: consideración en torno a la Resolución de la Dirección General de los Registros y del Notariado de 18 de febrero de 2009.

CAMACHO VARGAS, E./ JIMÉNEZ MATA, A. BENAVIDEZ SANTOS, D. Reflexiones sobre el derecho de familia costarricense, Universidad latina - EJC, Costa Rica, 2011.

DE LA MADRID, Mario. La cláusula de Elección del proceso judicial en los contratos internacionales, en RMDIPyC. № 28, mayo de 2011, pag. 57.

DREYZIN DE KLOR, A. El derecho internacional privado actual. Tomo I. Zavalia, Buenos Aires, 2015

- El derecho internacional de familia en la postmodernidad. EJC, Costa Rica, 2012.

- Restitución internacional de niñas y niños, Derecho de visita. EJC, Costa Rica, 2013.

INSTITUTO SUIZO DE DERECHO COMPARADO, The parental responsibility, child custody and visitation rights in cross-border separations, Lausanne, 2010.

GONZÁLEZ MÁRIN, Nuria. Luces y sombras convencionales y autónomas en materia de adopción internacional, en RMDIPyC. №24 junio de 2009.pág.159.

GRAHAM,JAMES.A. La restitución Internacional de menores en algunas tesis de los tribunales federales mexicanos, en RMDIPyC. N²7. Diciembre 2010. Pág.71

KEMELMAJER, A. /HERRERA, M./ LLOVERAS, N. Directoras., Tratado de Derecho de familia, Rubinzal Culzoni Editores, Argentina, 2014.

PEREZNIETO CASTRO, L. Derecho internacional privado. Parte general. 10a edición. Oxford, México, 2015.

- Dos mitos en el derecho internacional privado: la cláusula Calvo y la zona prohibida o zona restringida, en RMDIP. N¹, abril 1. 1997, pág. 5

- SILVA SILVA,J. Derecho Internacional Privado. Parte especial. Oxford, México, 2008.

- Y GRAHAM, La ley aplicable a la forma del matrimonio celebrado en el extranjero. Observaciones a la tesis CXIV/2006 de la Suprema Corte de Justicia de la Nación. En RMDIPYC. N²2. Octubre de 2008.pág.99.

- J. Tratado de Arbitraje Comercial internacional Mexicano. Limusa. México 2013 TENORIO GODINEZ, L./ TAGLE DE FERREYRA, G., La restitución internacional de la niñez, Ed. PORRÚA, MÉXICO, 2011.

VÁZQUEZ, Rodólfo, Derechos Humanos, una lectura liberal igualitaria, Ed. UNAMITAM-CONACYT. MÉXICO. 2015

WAITELET, Patrick, Private international law aspects of same-sex marriages and partnerships in Europe. Divided we stand? University of Liège. 


\section{B. Material normativo}

Convenios de las Conferencias Interamericanas de derecho internacional privado en materia de DIPr de Familia y de carácter procesal y la Convención de Normas Generales de 1979.

Convenios de ONU: Alimentos y el de reconocimiento de sentencias arbitrales de N.Y Convenios de La Conferencia de La Haya en materia de DIPr de Familia y sobre elección del foro.

La sistematización de estos Tratados internacionales puede verse en:

DREYZIN DE KLOR, A. /FERNÁNDEZ ARROYO, D., Derecho internacional privado argentino. Tratados en vigor y otros textos relevantes. Zavalía, Buenos Aires, 2009.

\section{Bibliografía}

1. ALEXY, Robert, Centro de Estudios Políticos y Constitucionales, Madrid, 2002.

2. GONZÁLEZ-BECERRIL, Juan Gabino, et al. (coords), Hitos demográficos del Siglo XXI: Migración Internacional, Universidad Autónoma del Estado de México, $2^{\mathrm{a}}$ edición, México, 2014, 377pp.

3. JAYME, Eric, Recueil des Cours, París, 2000. T.1. pag.25

4. Los Derechos Humanos en el derecho privado.

5. VON ÜVERBECK, A.E., Tirresistible extension de l'autonomie en droit international privé, Hommage Frar!fois Rigaux, Bruselas, 1993, pp. 619-636; 